\title{
IoT for wheel alignment monitoring system
}

\author{
Mohamad Hadi Sulaiman', Suhana Sulaiman², Azilah Saparon ${ }^{3}$ \\ ${ }^{2,3}$ Mechatronic and Intelligent System Research Group, Universiti Teknologi MARA (UiTM), Selangor, Malaysia \\ ${ }^{1,2,3}$ Faculty of Electrical Engineering, Universiti Teknologi MARA (UiTM), Selangor, Malaysia
}

\begin{tabular}{l}
\hline \hline Article Info \\
\hline Article history: \\
Received Aug 20, 2019 \\
Revised May 10, 2020 \\
Accepted Mar 4, 2021
\end{tabular}

Keywords:

Graphical user interface

IoT

Mobile application

System architecture

Wheel alignment

\begin{abstract}
A great deal of previous research into wheel alignment has focused on techniques of the alignment, which involve big, bulky and high cost to maintain. Even though several approaches are required, the works are tedious and only performed in spacious area and trained mechanics. IoT is the alternatives due to the evolution of smartphone with numerous sensors to support and assist the research and development for IoT applications in vehicles. In this work, smaller and portable wheel alignment monitoring system is introduced by using communication protocol between sensors, microcontroller and mobile phone application. Thus, graphical user interface (GUI) is utilized to the system via wireless communication technology using TCP/IP Communication Protocol. The system has been tested to suit the functioning architecture system for the wheel alignment to provide the user awareness on early detection of wheel misalignment. In addition, the application has been successfully integrated with Android mobile application via TCP/IP communication protocol and view the results in smart phone in real-time.
\end{abstract}

This is an open access article under the CC BY-SA license.

Corresponding Author:

Azilah Saparon

Faculty of Electrical Engineering

Universiti Teknologi MARA Shah Alam

40450 Shah Alam, Selangor, Malaysia

Email: azilah574@uitm.edu.my

\section{INTRODUCTION}

Wheel alignment for car's tyre plays an important role in ensuring the safety of a car on the road. Normally, a car owner will go to car workshop to check on the wheel alignment. It is known that car's wheel alignment can be monitored, adjusted and fixed inside the appointed car workshop. Conventional wheel alignment system is big, bulky and expensive. Furthermore, the driver needs to travel to workshop and the workshop normally operates during office hours.

To date, there are few methods in wheel alignment system including calibration using charge coupled device (CCD) [1]-[3] and 3D alignment [4], [5]. CCD device is known for its wheel alignment system using camera calibration and laser triangulation [1]. The arrangement is to achieve an accurate 3-D reconstruction of the wheel rim. Other method is vison- based comprises of machine vision and 3D vision with computer vision [6]-[8]. The later techniques is supported using an image acquisition system and camera calibration. All these techniques were adopted aiming to resolve the alignment of the wheels. However, the measurement techniques can be considered as big and bulky, not user friendly.

Hence, the wheel alignment monitoring system needs to be designed with functioning architecture system. This problem is potentially solved by using communication protocol between sensors, microcontroller and mobile phone that suit the functioning architecture system for wheel alignment which gives the user awareness on early detection of wheel misalignment. Internet of things (IoT) is adopted and 
potentially solved the issue since this method can be seen from any type applications [9] either for home [10] or vehicle security system [11]. Furthermore, the system can suit the functioning architecture system for wheel alignment. This alternative is possible for the car's driver to monitor the condition of the tyre whether it is aligned or misaligned to ensure the car's safety. In addition, user's wheel alignment monitoring systembased IoT is able to produce light and portable device for user to detect the tyre alignment inspection using smartphone. Although some google applications related to wheel alignment are available at Google playstore, there is no explanation or description on how the communication and system integration with sensors and its mobile application.

The significance of this study is to establish new method to measure wheel alignment by using time of flight/triangulation concept applied to ultrasonic/laser [12]-[14]. Apart from that, the new method can be applied into industrial application by implementing the graphical user interface (GUI) attached to the system thus reducing number of car accidents globally. Calculation for the best angle and position of transmitter/receiver is obtained based on the experiment [12]. The relationship between angle and position of transmitter/receiver is expected to be known. The application of wireless communication technology using $\mathrm{TCP} / \mathrm{IP}$ communication protocol is introduced to replace the wired communication for easy access in any field mostly in electronic devices.

This paper presents the development of IoT for wheel alignment monitoring system that is light and portable as well as user friendly. This development is equipped with communication protocol between sensors, microcontroller and mobile phone that suit with the functioning architecture system for wheel alignment. The concept of IoT for the wheel alignment provides an illustration of the model to elucidate the communication modules and the system architecture which could be applicable to any other applications. Finally, the product of the idea is deliberated in result and discussion section. The conclusion of this work is presented at the last section of this paper.

\section{METHOD FOR WHEEL ALIGNMENT MONITORING SYSTEM}

In this work, IoT is of interest because the technique of connecting everyday physical objects to the existing internet infrastructure [15]. Several works for ICT-based covers vehicle detection, road detection, lane detection, pedestrian detection, drowsiness detection and collison avoidances [16]. However, the current situation for the condition of a car has not been dictated for the road safety requirements. This has movitate the author to apply the IoT technique for the wheel alignment for the safety purposes. To illustrate, Figure 1 depicts an illustration of the model with the communication modules-microcontroller/microprocessor unit (MCU) and the system architecture respectively. There are four pairs of receivers/transmitters module which are responsible to measure the wheel alignment. Both infra-red receiver and transmitter are connected to ARM Cortex or AVR micro-controller that responsible for controlling the wheel alignment system. The micro-controller is programmed to transmit infra-red using infra-red transmitter component and receive infrared value using infra-red receiver component [8], [17], [18]. Then, the readings and measurements were transferred to the mobile application (Android/IOS) using wireless communication protocol (TCP/IP). Furthermore, TCP/IP communication protocol has been selected due to its flexibility, simplicity, scalability, and maintainability as well as cost effective [19], [20]. In addition, user-friendly and interactively graphical user interface (GUI) also assist the user with all the readings and measurements.

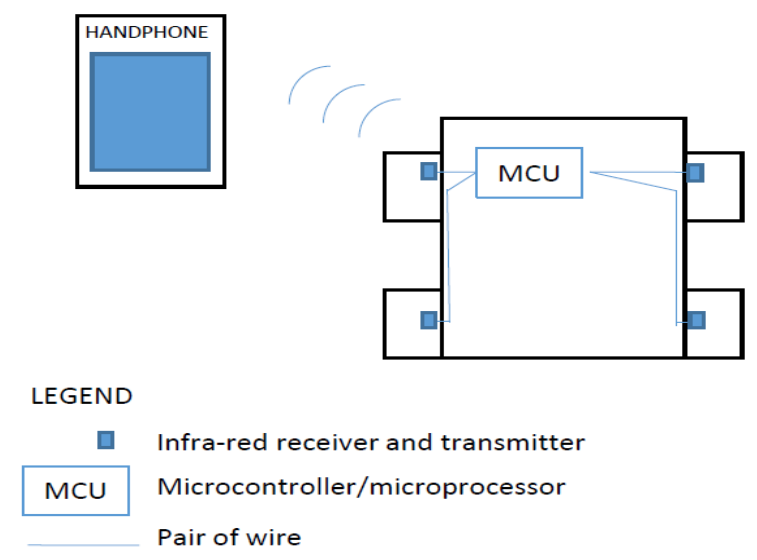

Figure 1. Illustration of wheel alignment system 
As far as data is concerned, the design variables (controlled factors) include reflection angles from light, infra-red transmitters and receivers, camber, caster, toe, mounting position of sensors, amount of infrared received and position between transmitters and receivers. The uncontrolled factors are noise, assembly error and sensors manufacturing errors. In this set up, infra-red receivers/transmitters were used to adopt the time of flight theory and modification on alignment calculation reading was made on calculation formula [21]-[23].

To implement the TCP/IP communication protocol, some requirements are considered. The use of hardware is required to control and to obtain reflection reading based on different angles, to connect base hardware with portable and viewable device and thus, we can have a product which is small in size. Hence, an analysis can be produced to meet the requirement.

On the software point of view, a system needs to be implemented on base hardware, which in this case is the Spark Core microcontroller. The system was programmed on the Spark Core microcontroller. Then it will control the logic flow to capture the reflection reading from the sensors, store inside the microcontroller and then passed to the viewable device. In order to make the product portable, small and low cost; the microcontroller is also being programmed to add the communication module between microcontroller and viewable device i.e. smart phone using TCP/IP communication protocol. The smart phone is chosen due to ease of access and capabilities to support various kinds of software modules and interactive graphical user interface (GUI). The TCP/IP communication protocol is chosen due to its reliability and performance compared to UDP protocol and availability of the open source libraries [24], [25].

Figure 2 elucidates the diagram of the wheel alignment system architecture for mobile application, ARM Cortex and the infra-red. ARM Cortex is the MCU for the system architecture. All of the data were stored inside the database and ready to be queried by the mobile application. The responsibility of the TCP server is to attend to any request from the TCP client which is the mobile application; and to send the data requested by the TCP client using TCP/IP (wireless) communication protocol.

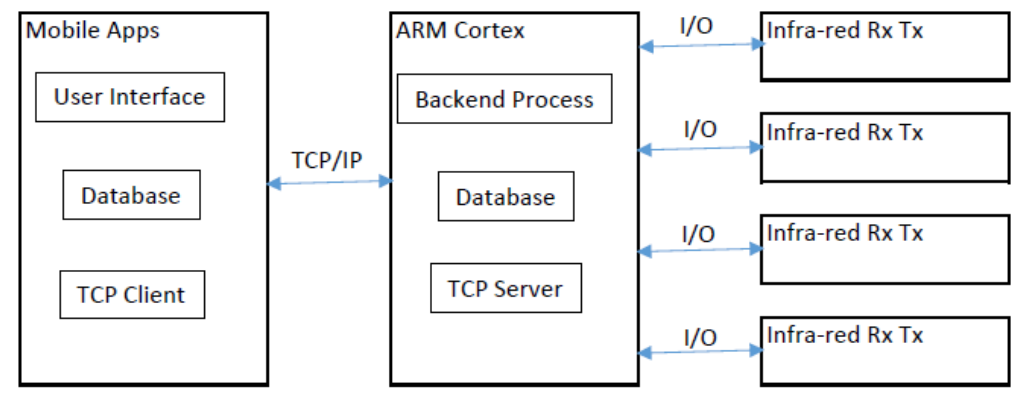

Figure 2. Diagram of wheel alignment system architecture

In the mobile application module, the data received from ARM Cortex requested by TCP Client were stored inside the database. The user interface sub-module will query real-time data from database and update to the graphical user interface to be seen by user. Also, there are an added notification processed to send notification to the user by email or short messaging service.

Figure 3 refers to software class diagram on microcontroller/microprocessor (ARM Cortex) application to operate and start up the Wi-Fi integrated circuit to detect the hot spot from the mobile device. The AlignmentProcess module will keep requested alignment reading from the IRReceiver using startIRReceiver function and commanding IRTransmitter to transmit pulse simultaneously. From the data received, the AlignmentProcess (control module) will send the date to CalcProcess module to calculate the alignment measurement. After the calculation process finished, the results will be stored inside the database using DBCore Controller module.

The DBCoreController module handle the data extracted from the calculation to store inside a database. DbWriter function is to write the data into the database file while dbReader is to read the data from database file and sent to any software client requester. BackupData function transfers accumulated data in database to a much larger database storage as requested by the software client through TCPServer.

The IOController module handle the interaction of I/O ports at the microcontroller. Each I/O pins have their own unique ID and it is very important for the other modules to identify which set of receivers or transmitters involved in the calculation. The TCPServer module responsible to initialize module for the server part in Client-Server Architecture for the future use. All these software modules aid the hardware integration to realize the parameters of the camber wheel alignment. 


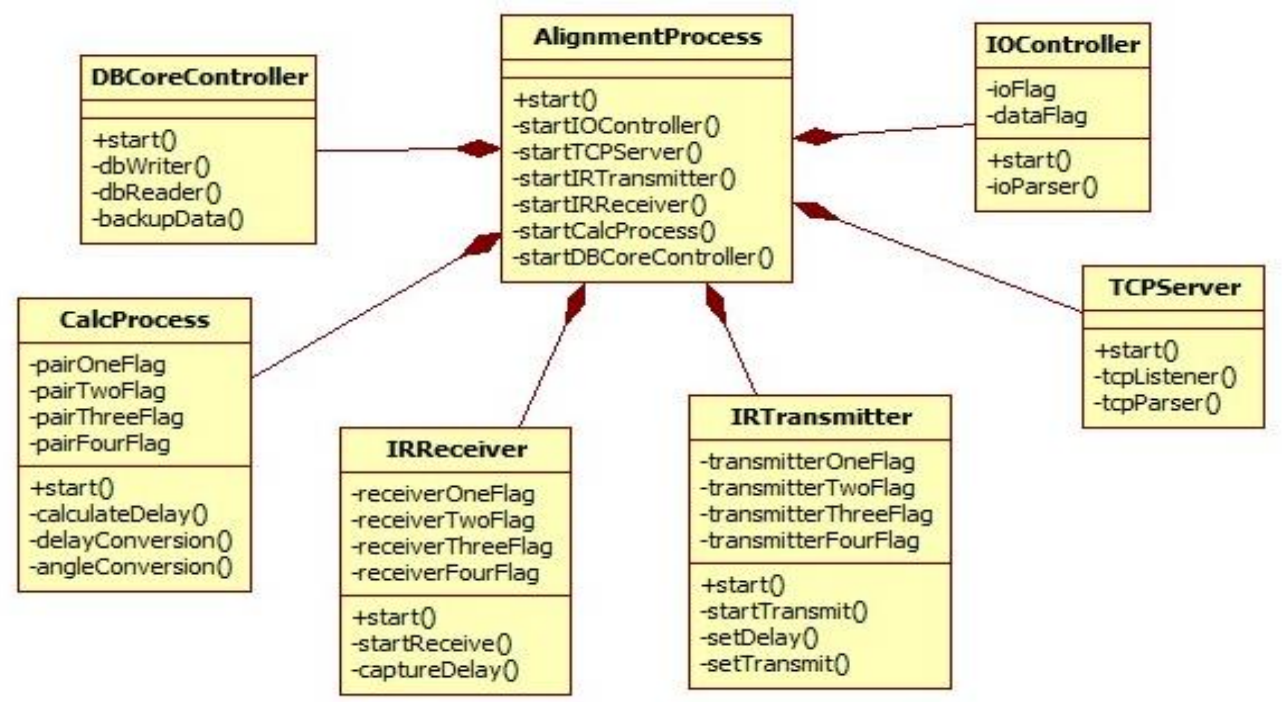

Figure 3. Software class diagram on microcontroller/microprocessor application

As shown in Figure 4, the diagram exhibits the software class diagram for the mobile application. This software development involves the overall control system and algorithm. Thus, when TCPClient module requests data in every one second delay from the TCPServer module in Figure 3, the data was controlled by AlignmentMonitoring module. The data received then will be stored inside the database by DBController module. The UserInterface module will always query for updated data inside the database and update the data to UserDashboard, GraphicalCar and LoginPage modules. The basic algorithm of the client side of the wheel alignment monitoring system using Android application and server side is coded with C++ compiled binary program.

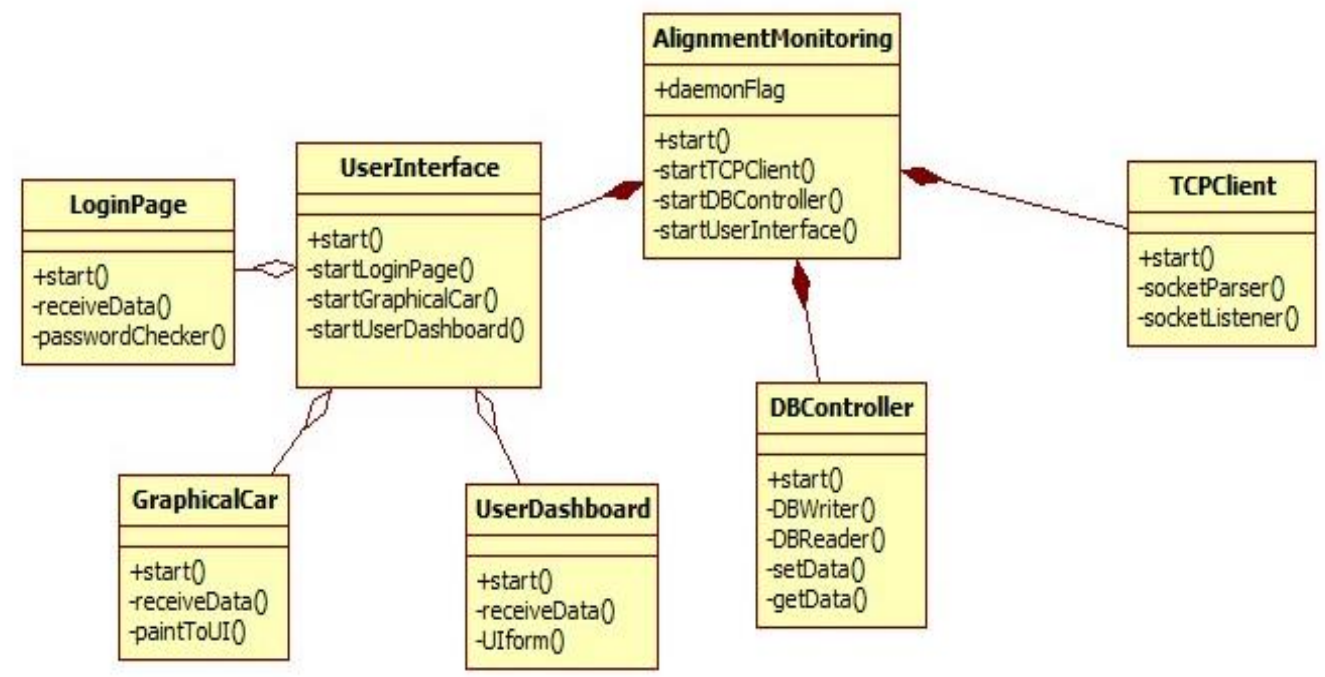

Figure 4. Software class diagram on mobile application

User interface for Android mobile application is user friendly and well-defined. The GraphicalCar module in Figure 4 responsible to view a car diagram from top view to make it understandable in the user's perspective. It consists a picture of a car with four tyres and rectangle view on each of the tyre representing the alignment condition and the value of the infrared alignment parameter. The alignment text and value of alignment parameter will be changed in real-time depends on the value received by the TCPClient module and microcontroller reading on the alignment. 


\section{RESULTS AND DISCUSSION}

This study set out with the aim of assessing the importance of IoT for wheel alignment monitoring system. Figure 5 depicts the prototype set-up for the integration of GUI and the car wheels and the mobile applications. The system comprises of smartphone for the GUI, the toy car's tyre was set up to test the measurement of wheel alignment using time of flight/triangulation concept to check the alignment. The hardware for the four pairs of receivers/transmitters module which are responsible to measure the wheel alignment is also included. Both infra-red receiver and transmitter are connected to ARM Cortex or AVR micro-controller that responsible for controlling the wheel alignment system.

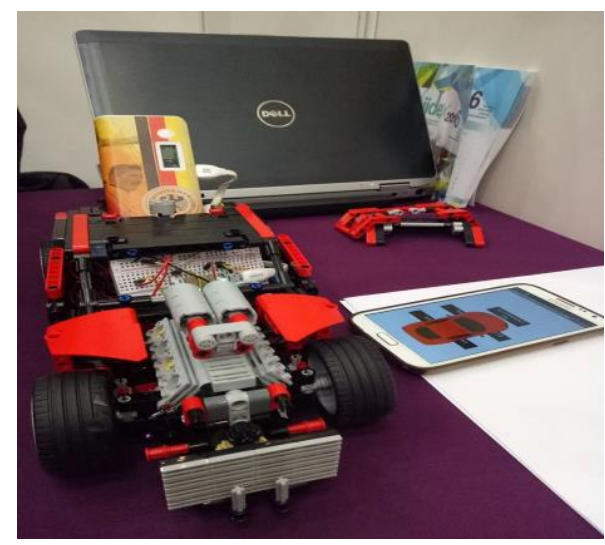

Figure 5. Prototype set-up for the wheel, GUI and mobile applications

Figure 6 displays the result for design for the mobile application (Android/IOS) graphical user interface (GUI) module. In the GUI interface, there is graphical car with 4 wheel images button to provide user friendly experience to the user. Once the wheel image button is clicked, a pop-up will show the alignment information. On the right side there will be an additional information of user and the vehicle. User can use email or SMS to send notification when the alignment need to be adjusted. Vehicle information such as model, colour, year of manufacture and engine capacity can also be added to Car Info. This can be useful in future prospect when all the vehicles information or updates are centralized globally.
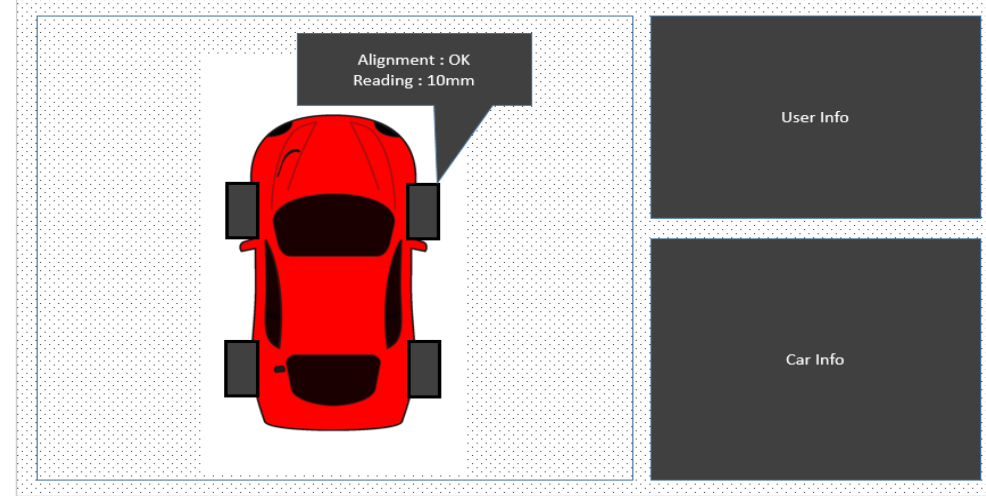

Figure 6. Overview of graphical user interface on mobile application

As mentioned previously, the connection between Android mobile application and microcontroller application were prepared wirelessly. The Android mobile application is using Samsung Galaxy Note 2 as its mobile application medium. The TCP client module will start to search for TCP server designated IP and port. The programming for the TCP server designated IP and port were coded with the Java code. Once the TCP client connected with the TCP server application on the microcontroller, the UserInterface module were established, the graphical view of car, alignment condition and alignment infra-red value will appear as displayed in Figure 7. 
As mentioned previously, the system has been tested to suit the functioning architecture system for the wheel alignment. Figures 7,8 and 9 are referred to the recorded work on the real tyre during the experimental set up of integrating wheel alignment monitoring system using microcontroller with the Android mobile application.

As exhibited in Figure 7, the Spark Core microcontroller circuit on the breadboard is powered by normal power bank to produce $5 \mathrm{~V}$ and 1 A current rating. After the microcontroller is turned on, the light indicator on Spark Core will turn from green to blue colour indicating that the module is already connected on the designated Wi-Fi network, in this case the smart phone is used for the tethering mobile hotspot to allow Wi-Fi connection from the microcontroller. Hence, both microcontroller and Android mobile application were in the same Wi-Fi network. In the background, TCP Server of the microcontroller application is already running and attending to connection from the TCP client from the mobile application. In this set up, the breadboard with microcontroller is placed inside the dashboard of car.

Figure 8 indicates the two pair of wires from the breadboard both connected with infra-red transmitter and infra-red receiver respectively. Both infra-red transmitter and infra-red receiver were installed throughout the car from the dashboard to the front left side of the tyre location. With the assistance of the appointed mechanic, the wire was installed from the dashboard to the mounting location by using the space on the hole below the dashboard.

Figure 9 shows the pair of wire as discussed in Figure 8 is coupled with a pair of infra-red transmitter and receiver. At this point, the transmitter and receiver is mounted near to the absorber mount. Then, the angle and distance parameter are measured manually. The design of the mounting specifically to mount these infra-red transmitter and receiver can further be improved in the future.

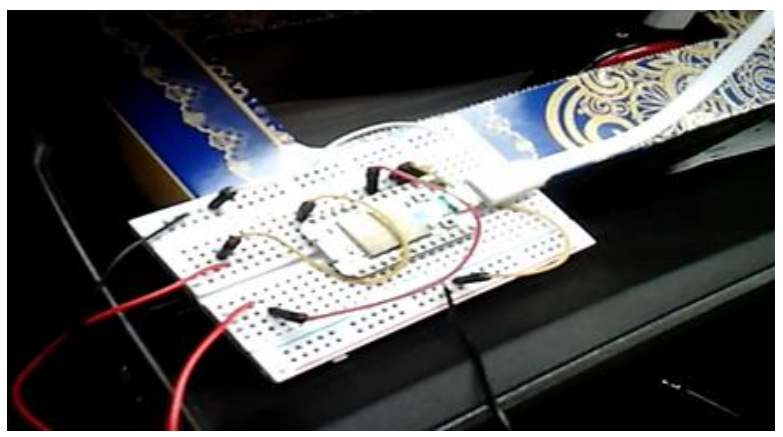

Figure 7. Microcontroller setup connected with $5 \mathrm{~V}$ input power bank

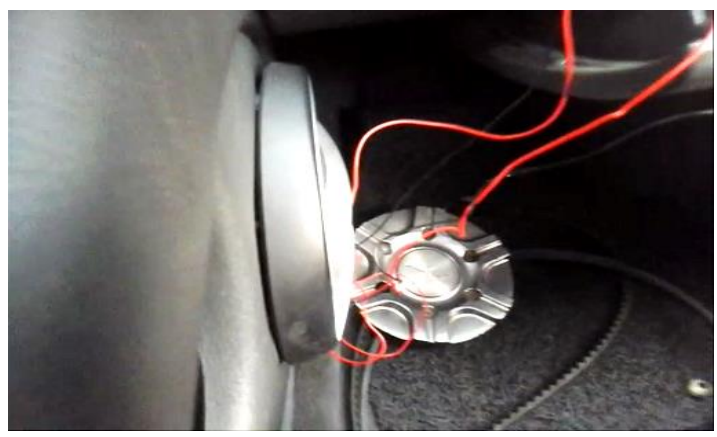

Figure 8. Wire connection from microcontroller to the left side of car's tyre

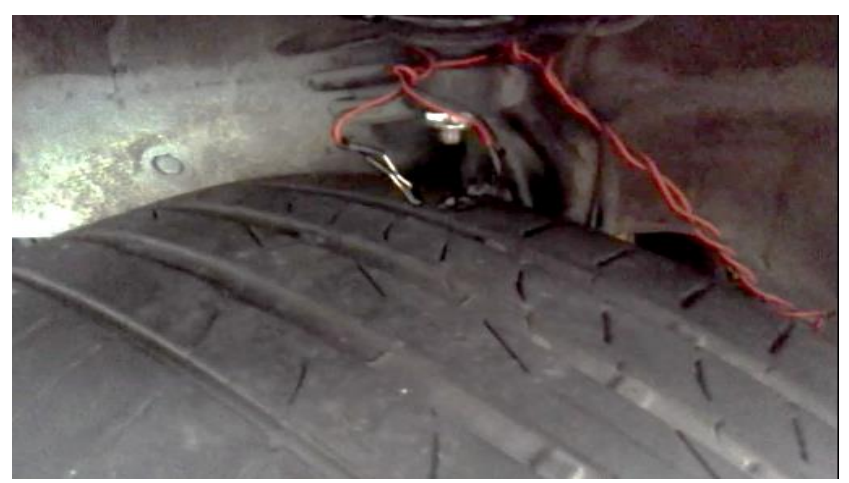

Figure 9. From wire connection to the infra-red receiver and transmitter with temporary mount

When the Android mobile application starts running and displaying the User Interface, the alignment condition and value is observed when the connection between TCP server and TCP client were established. The parameter setup for the mobile application has been set to indicate wheel alignment is normal and good when the range value of 20 to 150 with the initial distance of tyre of $15 \mathrm{~mm}$, angle of infra-red receiver and transmitter of $60^{\circ}$ were detected. 
The result is displayed in Figure 10 where the infra-red reading is out of reach as the angle of tyre intentionally adjusted to out-of-bound angle. Hence, the alignment monitoring GUI indicates that the alignment is not ok. This angle of tyre is intentionally adjusted to the misalignment condition with the assistance of appointed mechanic.

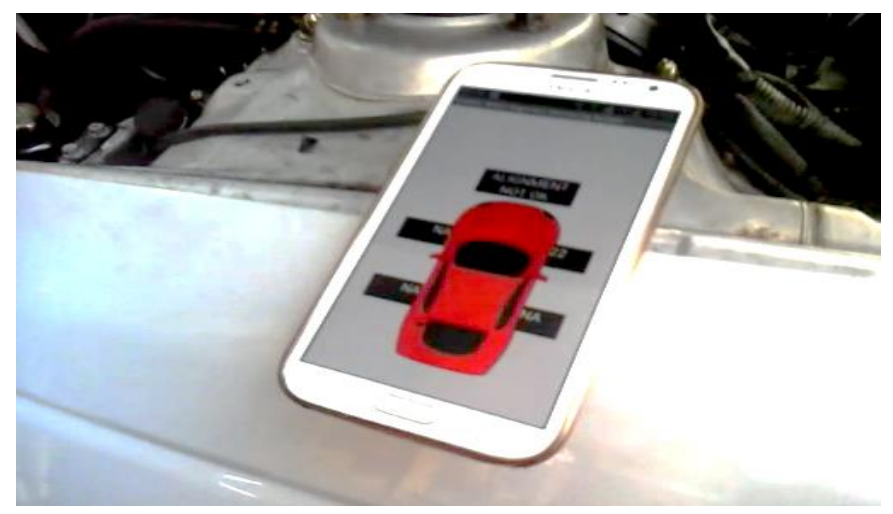

Figure 10. The android mobile application connected wirelessly with microcontroller

Figures 11 and 12 exhibit the result of wheel alignment monitoring system using microcontroller application has been integrated with Android mobile application via TCP/IP communication protocol and view the findings in smart phone in real-time. The outcome reveals that both the hardware module and the software for the TCP/IP communication protocol has been successfully implemented and experimentally verified.

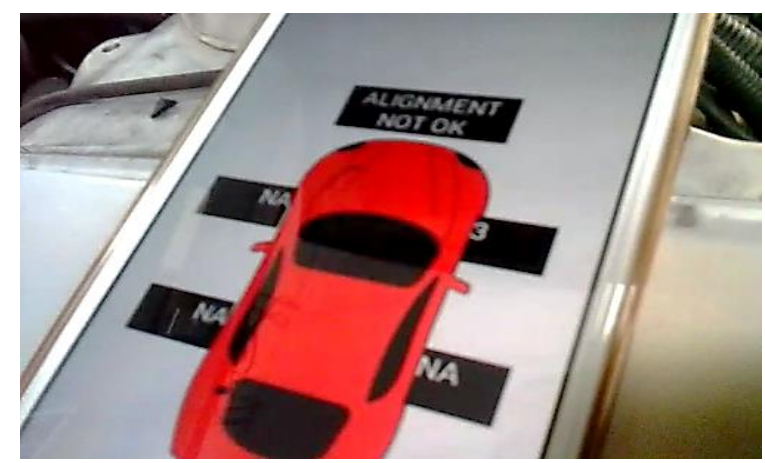

Figure 11. Result of android mobile application indicating the alignment not ok

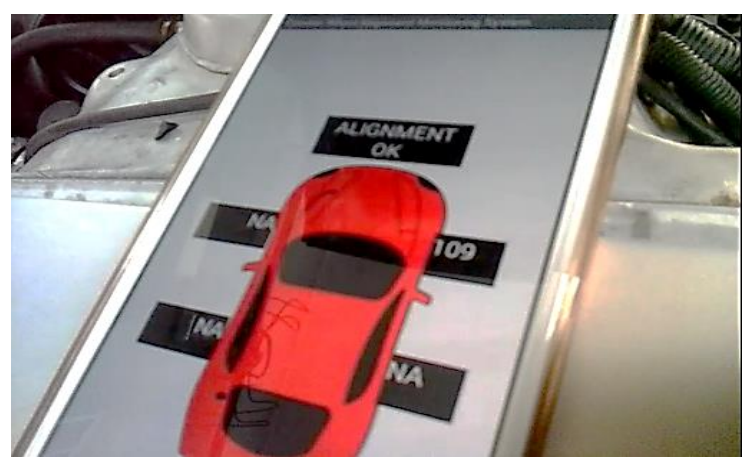

Figure 12. Result of android mobile application indicating the alignment ok

\section{CONCLUSION}

To conclude, IoT for wheel alignment monitoring that is light and portable as well as user friendly is realized. The application is equipped with communication protocol between sensors, microcontroller and mobile phone that suit the functioning architecture system for wheel alignment. The product of this technique is the wheel alignment monitoring system using microcontroller application has been successfully integrated with Android mobile application via TCP/IP communication protocol and view the results in smart phone in real-time. The experimental set up was performed in the workshop with the assistance of the appointed mechanic.

\section{ACKNOWLEDGEMENTS}

The authors gratefully acknowledge Institute of Research Management \& Innovation (IRMI), UiTM Shah Alam, Selangor in providing fund for this work under project no: 600-RMI/DANA 5/3/LESTARI (101/2015). 


\section{REFERENCES}

[1] P. P. J. Senjalia, "Measurement of wheel alignment using Camera Calibration and Laser Triangulation," 2013 Nirma University International Conference on Engineering (NUiCONE), 2013, pp. 2-6, doi: 10.1109/NUiCONE.2013.6780177.

[2] R. E. Anna, "A Method for Image Processing and Distance Measuring Based on Laser Distance Triangulation," 2013 IEEE 20th International Conference on Electronics, Circuits, and Systems, ICECS2013, 2013, pp. 1-3, doi: 10.1109/ICECS.2013.6815509.

[3] J. W. M. S. Park, "Experimental study on camera calibration and pose estimation for the application to vehicle's wheel alignment," 2006 SICE-ICASE International Joint Conference., 2006, pp. 2952-2957, doi: 10.1109/SICE.2006.314911.

[4] G. Smith, "CCD Alignment Systems VS. 3D Alignment Systems," [Online]. Available: http://www.gregsmithequipment.com/CCD-Vs-3D-Alignment-Systems.

[5] J. Ludwig, "Wheel Alignment in Endicott, NY," precision automotive service, [Online]. Available: http://www.precisionautomotiveny.com/wheel-alignment/.

[6] Y. G. W. Li, "Research on the machine vision system for vehicle four-wheel alignment parameters," Proceedings of the 30th Chinese Control Conference, 2011, pp. 3192-3195.

[7] X. K. Hu, "Study on calibration methods of two cameras in vehicle's four-wheel alignment based on 3D vision," Proc.-2010 3rd Int. Congr. Image Signal Process. CISP 2010, 2010, pp. 414-416, doi: 10.1109/CISP.2010.5647994

[8] D. Wang and Y. Tu, "An approach to measuring vehicle four-wheel alignment parameters based on computer vision," 2007 8th International Conference on Electronic Measurement and Instruments, 2007, pp. 391-394, doi: 10.1109/ICEMI.2007.4351065.

[9] R. Amine and A. Oumnad, "Internet of Things: Surveys for measuring human activities from everywhere," International Journal of Electrical and Computer Engineering (IJECE), vol. 7, no. 5, pp. 2474-2482, 2017.

[10] T. Gunawan, I. R. H. Yaldi, M. Kartiwi, and H. Mansor, "Performance evaluation of smart home system using internet of things," International Journal of Electrical and Computer Engineering (IJECE), vol. 8, no. 1, pp. 400-411, 2018.

[11] S. Palaniapan and M. A. Kollathodi, "Real time implementation of embedded devices as a secuiryt system in intelligent vehicles connected via Vanets," International Journal of Electrical and Computer Engineering (IJECE), vol. 9, no. 6, pp. 4788-4797, Dec. 2019.

[12] M. H. Sulaiman, A. Saparon, and S. Sulaiman, "Wheel alignment parameters based on laser angle of reflection with TCP/IP protocol," 2016 International Conference on Advances in Electrical, Electronic and Systems Engineering, ICAEES 2016 - Putrajaya, Malaysia, 2016, pp. 233-236.

[13] J. Phillips, R. Green, and M. Alam, "Time-of-Flight Distance Measurements Using Smart Phones," 2014 IEEE International Conference on Mobile Services, 2014, pp. 153-154, doi: 10.1109/MobServ.2014.31.

[14] S. Lanzisera, D. Zats, and K. S. J. Pister, "Radio frequency time-of-flight distance measurement for low-cost wireless sensor localization," IEEE Sensors Journal, vol. 11, no. 3, pp. 837-845, 2011, doi: 10.1109/JSEN.2010.2072496.

[15] I. S. J. Wahlström, "Smartphone-Based Vehicle Telematics: A Ten-Year Anniversary," IEEE Transactions on Intelligent Transportation Systems, pp. 2802-2824, 2017.

[16] C. Bila, F. Sivrikaya, M. A. Khan, and S. Albayrak, "Vehicles of the Future: A Survey of Research on Safety Issues," IEEE Transactions on Intelligent Transportation Systems, pp. 1046-1064, 2017, doi: 10.1109/TITS.2016.2600300.

[17] P. B. Q. Liu, "Ultrasonic Distance Measuring System Without Blind Area," 2016 IEEE International Conference on Signal and Image Processing (ICSIP), 2016, pp. 626-629.

[18] S. Zhang, "Research of ultrasonic distance measurement device," 2010 6th International Conference on Wireless Communications Networking and Mobile Computing (WiCOM), 2010, pp. 2-4, doi: 10.1109/WICOM.2010.5601109.

[19] T. Araari, I. Jaziri, L. Charaabi, and K. Jelassi, "DC motor control based on embedded Linux," 2014 International Conference on Electrical Sciences and Technologies in Maghreb (CISTEM), 2014, pp. 1-6, doi: 10.1109/CISTEM.2014.7076921.

[20] A. Nakanishi, K. Hatayama, T. Onoduka, and T. Kimura, "An Embedded System of TCP / IP Communication by Using FPGA," 2015 IEEE 4th Global Conference on Consumer Electronics (GCCE), 2015, pp. 489-492, doi: 10.1109/GCCE.2015.7398630.

[21] J. Seiter, M. Hofbauer, M. Davidovic, S. Schidl, and H. Zimmermann, "Correction of the temperature induced error of the illumination source in a time-of-flight distance measurement setup," 2013 IEEE Sensors Applications Symposium Proceedings., 2013, pp. 84-87, doi: 10.1109/SAS.2013.6493562.

[22] S. Schwarz, M. Sjöström, and R. Olsson, "A weighted optimization approach to time-of-flight sensor fusion," IEEE Trans.Image Process., vol. 23, no. 1, pp. 214-225, 2014.

[23] X. C. X. Chen and C. W. C. Wu, "Ultrasonic Distance Measurement Based on Infrared Communication Technology," 2009 Third International Symposium on Intelligent Information Technology Application, 2009, vol. 1, pp. 0-3, doi: 10.1109/IITA.2009.243.

[24] F. Al-Dhief, N. Sabri, and M. Albadr, "Performance Comparison between TCP and UDP Protocols in Different Simulation Scenarios," International Journal of Engineering \& Technology, vol. 4, no. 36, pp. 172-176, 2018. 
[25] S. Suherman and M. Al-Akaidi, "A review on transport layer protocol performance for delivering video on an adhoc network," IOP Conference Series: Materials Science and Engineering, vol. 237, 2017, doi: 10.1088/1757899X/237/1/012018.

\section{BIOGRAPHIES OF AUTHORS}
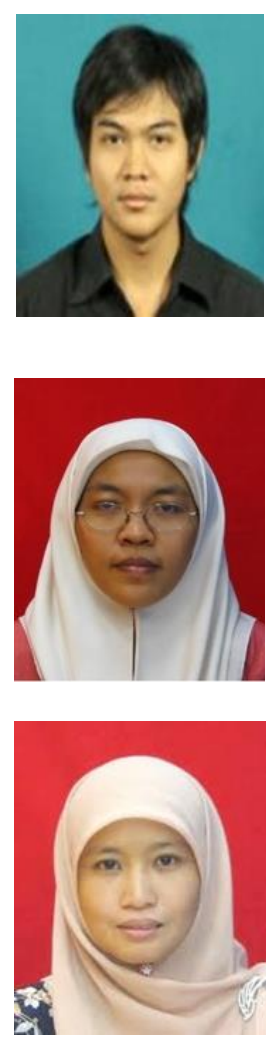

Mohammad Hadi Sulaiman received his B.Eng (Hons) in Electrical Engineering, in 2012 from Multimedia University and M.Sc in Electrical Engineering in 2019 from Universiti Teknologi MARA Shah Alam, Selangor, Malaysia. Currently, he is working as Senior Software Engineer. His research interests include Software Development, Artificial Intelligence and Embedded System. Apart from that, he established his own company known as Umtiaz Innotech Sdn Bhd which offers hardware and software products. The latest product the company has developed was web application development known as LigaKita.my which serves as a gaming platform for Malaysian footballers. He also ventures in e-commerce platform to sells football merchandises. His latest research was the development of wheel alignment monitoring system prototype.

Suhana Sulaiman received her B.Sc in Electrical Engineering, from University of Utah, Salt Lake City, USA in 1996 and M.Sc in Microelectronics from University of Newcastle Upon Tyne, UK in 2002. Her Ph.D degree, in Electrical Engineering from Universiti Teknologi MARA, Shah Alam Selangor, Malaysia in 2013. Currently, she is a senior lecturer in Faculty of Electrical Engineering, UiTM Shah Alam, Selangor. Her research interests include RFIC, microelectronics, electronics circuit and system and microfabrication.

Azilah Saparon, is an Associate Professor at Faculty of Electrical Engineering, UiTM Shah Alam, Selangor, Malaysia. She received her B.E.E from Gannon University, Erie, Pennsylvania, U.S.A in 1989, M.Sc in Electrical Engineering from Washington State University at Pullman,U.S.A in 1995 and a Ph.D in Electronic and Electrical Engineering from Loughborough University, Leicestershire, U.K 2006. Her research interests include reconfigurable system, micro-computer architecture and video coding. 